\title{
Renouvellement et institutionnalisation des centrales syndicales au Venezuela sous Chávez $(2001-2011)$
}

Renewal and institutionalization of trade unions in Venezuela's Chavez (2001 2011)

Renovación e institucionalización de las centrales sindicales en la Venezuela de Chávez (2001 - 2011)

\section{Thomas Posado}

\section{OpenEdition}

\section{Journals}

Édition électronique

URL : https://journals.openedition.org/ideas/832

DOI : $10.4000 /$ ideas.832

ISSN : 1950-5701

\section{Éditeur}

Institut des Amériques

\section{Référence électronique}

Thomas Posado, «Renouvellement et institutionnalisation des centrales syndicales au Venezuela sous Chávez (2001 - 2011) », IdeAs [En ligne], 5| 2015, mis en ligne le 15 juin 2015, consulté le 18 octobre 2022. URL : http://journals.openedition.org/ideas/832 ; DOI : https://doi.org/10.4000/ideas 832

Ce document a été généré automatiquement le 18 octobre 2022.

\section{cc) (†)}

Creative Commons - Attribution - Pas d'Utilisation Commerciale - Pas de Modification 4.0 International - CC BY-NC-ND 4.0

https://creativecommons.org/licenses/by-nc-nd/4.0/ 


\section{Renouvellement et institutionnalisation des centrales syndicales au Venezuela sous Chávez $(2001$ - 2011)}

Renewal and institutionalization of trade unions in Venezuela's Chavez (2001 2011)

Renovación e institucionalización de las centrales sindicales en la Venezuela de Chávez (2001 - 2011)

Thomas Posado

\section{Introduction}

Le marché du travail en Amérique latine a connu de profondes mutations pendant les trente dernières années. Ces transformations n'ont pas été sans conséquences sur la représentation du monde du travail. Au Venezuela, des réformes d'inspiration néolibérale ont précédé l'élection d'Hugo Chávez en 1998. La polarisation autour de ce gouvernement a entraîné un dynamisme rarement constaté dans les recompositions syndicales. En dix ans, trois confédérations syndicales se sont succédées à la tête du mouvement ouvrier. La Confédération des travailleurs du Venezuela (CTV), la centrale syndicale historique, organise des élections en octobre 2001. La nouvelle direction adopte une stratégie en rupture à l'égard du gouvernement d'Hugo Chávez. Ce dernier soutient en avril 2003 la création d'une nouvelle centrale syndicale : l'Union nationale des travailleurs (UNT). Les tensions à l'intérieur de cette structure naissante aboutissent en novembre 2011 à la fondation d'une confédération: la Centrale bolivarienne socialiste des travailleurs de la ville, de la campagne et de la pêche (CBST$\mathrm{CCP})^{1}$. La direction du mouvement syndical vénézuélien traditionnel a été renversée en relation avec la consolidation du gouvernement d'Hugo Chávez par l'action conjointe 
du gouvernement et de larges secteurs de travailleurs. De quoi ces changements dans l'action du mouvement politique impulsé par le président Hugo Chavez sur le mouvement ouvrier vénézuélien sont-ils révélateurs?

Ces recherches sont issues d'un doctorat en cours portant sur les recompositions syndicales dans le Venezuela contemporain. Nous nous proposons dans cet article d'analyser les trajectoires syndicales des dirigeants appartenant aux trois groupes suivants : le Comité exécutif de la CTV élu en 2001, le Comité coordinateur national provisoire de l'UNT ayant pris ses fonctions en 2003 et la Coordination nationale de la CBST-CCP créée en 2011.

Terrain et méthode

Dix critères ont été pris en compte pour l'ensemble des 59 dirigeants syndicaux de notre sélection. Ces dix critères sont : le secteur syndical du moment ; la région du moment ; le secteur syndical du premier mandat syndical ; la région du premier mandat syndical ; la date du premier mandat syndical ; le cheminement politique ; l'origine socio-professionnelle des parents ; l'année de naissance ; le dernier diplôme obtenu et une auto-évaluation sur un axe gauche-droite allant de 0 à 10 , indicateur critiquable en soi mais qui constitue un indice de l'auto-représentation relative des acteurs. L'enquête a été menée pendant l'été 2013 et la méthode privilégiée est celle d'entretiens semi-directifs donnant une dimension qualitative aux données recueillies. Nous avons pu interroger un peu moins de la moitié de l'échantillon de la CTV, un tiers de celui de l'UNT et un peu plus de la moitié de celui de la CBST-CCP et obtenu, dans la mesure du possible, des données sur les dirigeants manquants de manière indirecte par le biais de leurs collègues afin de rendre les résultats plus représentatifs. Nous proposons ici une première analyse du travail obtenu.

\section{La CTV, organe du parti social-démocrate dominant avant Chávez}

3 La CTV a été fondée en 1936 et a été la courroie de transmission du parti socialdémocrate qui dominait la vie politique vénézuélienne avant Chávez, Action démocratique $(\mathrm{AD})$, du rétablissement de la démocratie en 1958 jusqu'à 2001. Face à la crise économique survenue au Venezuela à partir de 1983 et à la mise en place des réformes d'inspiration néo-libérale, la CTV s'avère incapable d'organiser le mécontentement croissant des classes populaires vénézuéliennes ni de se mobiliser pour défendre leurs intérêts. Le président de la CTV est brièvement incarcéré pour des soupçons de corruption en 1992. Le taux de syndicalisation baisse de $26,4 \%$ en 1988 à 13,5\% en 1995. La direction de la CTV refuse en 1994 la tenue d'élections internes pour le $11^{\mathrm{e}}$ Congrès de 1995, mais s'engage à leur organisation dès 1999 (Ellner S., $1995: 149$ ). Au moment de l'investiture du président Chávez en février 1999, un sondage révèle que les syndicats jouissent d'un taux d'opinions favorables de $21,9 \%$ faisant des ces institutions les plus impopulaires du pays (Gil Yepes J. A., $2007: 282$ ). En décembre 2000, le gouvernement convoque un référendum portant sur la rénovation intégrale des directions syndicales. Le résultat de ce référendum est positif avec $62,02 \%$ des suffrages exprimés, malgré un taux de participation plutôt faible $(23,5 \%)^{2}$. Des 
élections syndicales générales sont organisées le 25 octobre 2001. La coalition des opposants à Chávez, le Front uni des travailleurs (FUT), dirigée par l'ancien député AD, Carlos Ortega, l'emporte largement avec $57 \%$ des suffrages et obtient 10 sièges au Comité exécutif. Son concurrent immédiat, Aristóbulo Istúriz, représentant les partisans du gouvernement dans le Front bolivarien des travailleurs (FBT) ne parvient à regrouper que $16 \%$ des voix, soit deux sièges au Comité exécutif. Hors de ces deux coalitions, une liste dissidente de gauche «Nouveau syndicalisme/mouvement premier mai» (NS / MPM) obtient $11 \%$ des votes, soit deux sièges. Les trois propositions plus minoritaires obtiennent un siège chacun (Alliance Syndicale Indépendante (ASI), $6 \%$; Front constitutionnel des travailleurs (FCT), $5 \%$ et Front bolivarien des travailleurs-200 (FBT-200), $4 \%$ ). Malgré la surveillance du Conseil national électoral, tout laisse penser que des fraudes semblent avoir eu lieu en faveur du président élu sans toutefois être de nature à inverser l'ordre d'arrivée des candidats (Iranzo C., 2002:60). Dans la partie suivante nous étudierons plus en détail la trajectoire des 17 personnes faisant partie du Comité exécutif.

\section{Comité exécutif de la CTV élu en octobre 2001}

\begin{tabular}{|l|l|l|}
\hline & Prénom Nom & Liste \\
\hline 1 & Carmen Omaira Arismendi & FUT \\
\hline 2 & Froilán Barrios & FCT \\
\hline 3 & Pablo Castro & FUT \\
\hline 4 & Manuel Cova & FUT \\
\hline 5 & Aristóbulo Istúriz & FBT \\
\hline 6 & Pedro Arturo Moreno & FUT \\
\hline 7 & Pedro Natera & FUT \\
\hline 8 & Carlos Navarro & ASI \\
\hline 9 & Carlos Ortega & FUT \\
\hline 10 & Adolfo Padrón & FUT \\
\hline 11 & Rodrigo Penso & NS/MPM \\
\hline 12 & Ramón Petit & FUT \\
\hline 13 & Jesús Ramírez & FBT \\
\hline 14 & Alfredo Ramos \\
\hline 15 & Angel Rodríguez & Reyna Sequera \\
\hline 16 MPM \\
\hline
\end{tabular}




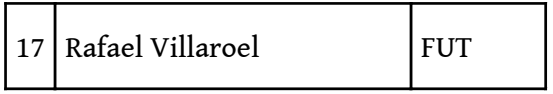

SOURCE : CTV

4 Nous distinguons dans le tableau précédent trois groupes : les dirigeants $(1,4,7,9,10$, $12,13,17)$, la gauche $(2,3,6,11)$ et les démissionnaires $(5,8,14,15,16)$. Les dirigeants sont tous issus de la liste gagnante du FUT, membre du parti social-démocrate AD, à l'exception d'un d'entre eux (12), qui est membre de l'autre parti dominant (le COPEI ${ }^{3}$, le parti social-chrétien). Au moment de l'élection en 2001, les dirigeants habitent tous à Caracas où ils gèrent les principales fédérations du pays: pétrole (9), santé (7), construction (4) ou encore éducation (13). Dans l'élection qui a lieu en octobre 2001, ils remportent les trois principaux postes de la centrale syndicale, à savoir : la présidence (9), le secrétariat général (4) et la trésorerie (12). Ils ont tous des origines populaires et doivent leur position sociale à $\mathrm{AD}$ qui les a promus à la tête du champ syndical national. Ils partagent des positions de centre-gauche (centre-droit pour le représentant du COPEI) et manifestent une hostilité ouverte à l'égard du gouvernement d'Hugo Chávez. Cette animosité les poussera à faire partie des tentatives de renversement d'Hugo Chávez au moment du coup d'État militaire d'avril 2002 et de l' «insurrection des gérants $»^{4}$ de décembre 2002 à février 2003. L'échec de cette stratégie va atomiser leur positionnement politique: l'un prend la route de l'exil (9), les autres continuent à diriger une CTV affaiblie en s'émancipant des anciens partis dominants $(1,4,12)$, et d'autres encore restent fidèles à $\mathrm{AD}$ tout en s'éloignant de la direction de la confédération (7 décédé depuis, 10, 13,17).

5 L'aile gauche de la représentation syndicale est issue pour deux d'entre eux de la liste victorieuse sur laquelle ils avaient été cooptés $(3,6)$ et deux autres élus sur des listes de gauche à l'audience plus réduite $(2,11)$. Ils proviennent tous des petits partis de la gauche vénézuélienne, qui étaient marginaux jusqu'à l'émergence d'Hugo Chávez : MIR, MAS $(2,11), B R(6), \operatorname{MEP}(3)$. Ils n'ont pas de syndicat de base suffisamment puissant, à une exception près (3), mais obtiennent des sièges dans la direction nationale de la Centrale syndicale pour mener leur combat politico-syndical. Les origines sociales de ces leaders sont plus élevées que celles du groupe précédent : les quatre dirigeants se déclarent provenir des classes moyennes $(2,3,6)$ ou d'un milieu semi-oligarchique (11): fils de professeurs ayant fondé leur propre collège (2), petit commerçant (6), propriétaire terrien d'un domaine plus ou moins grand $(3,11)$. Ils partagent la même opposition au gouvernement d'Hugo Chávez, même si certains d'entre eux ont critiqué le caractère hasardeux de la stratégie du blocage de l'économie de décembre 2002 à février 2003. Ils sont les initiateurs du Mouvement Solidarité au Travail ${ }^{5}$ en 2009 puis du Front autonome de défense de l'emploi, du salaire et du syndicat (FADESS) en 2011, composé en outre par des courants déçus du chavisme.

6 Les démissionnaires sont plus hétérogènes. Bien qu'ils soient élus, ils n'ont jamais siégé au Comité exécutif de la CTV. Celui-ci n'était pas à leurs yeux légitime au vu des tricheries observées lors des élections. Deux d'entre eux représentent la liste explicitement soutenue par le gouvernement $(5,15)$ et trois sont issus de petites listes minoritaires $(8,14,16)$. Leurs parcours montrent la difficulté du champ syndical à conserver des cadres qui cherchent à intégrer des positions politiques. Les dirigeants syndicaux "chavistes" ont été appelés à de hautes fonctions politiques: l'un est devenu gouverneur d'un État à l'Est du pays (5), l'autre a été brièvement Ministre de 
l'Énergie vers la fin de l'année 2009 et dirige depuis quelques mois un programme social du gouvernement (15). Concernant les trois autres, l'une dirige un parti politique marginal (16), l'autre est devenu maire d'une des plus grandes villes du pays (14), le troisième, secrétaire général sortant de la CTV a fondé une centrale syndicale autonome faisant partie du MSL puis du FADESS. Cela illustre l'atomisation du mouvement syndical d'opposition évoquée précédemment.

\section{L'UNT, centrale éphémère renouvelant les directions syndicales}

7 Une nouvelle centrale syndicale, l'UNT, est fondée en avril 2003 juste après l' "insurrection des gérants" de décembre 2002 et occupe rapidement une position hégémonique dans le champ syndical. Dès le lendemain de sa création, la structure créée ex-nihilo annonce près d'un million d'adhérents. Une direction collégiale se met en place sur des rapports de forces supposés : 21 coordinateurs nationaux sont désignés sans hiérarchie entre eux.

Comité coordinateur national provisoire de l'UNT installé en avril 2003

\begin{tabular}{|l|l|}
\hline & Prénom, Nom \\
\hline A & Juan Cahuao \\
\hline B & Orlando Castillo \\
\hline C & Orlando Chirino \\
\hline D & Juan Crespo \\
\hline E & Richard Gallardo \\
\hline F & José Gil \\
\hline G & Marcos Mariño \\
\hline H & Marcela Máspero \\
\hline I & José Mora Siguaraya \\
\hline J & Henry Navas \\
\hline K & Jesús Noriega \\
\hline L & Nelson Nuñez \\
\hline M & Joaquin Osorio \\
\hline N & Stalin Pérez Borges \\
\hline O & Eduardo Piñate \\
\hline
\end{tabular}




\begin{tabular}{|l|l|}
\hline P & Juan Reyes \\
\hline Q & Franklin Rondón \\
\hline R & Rafael Rosales \\
\hline S & Francisco Torrealba \\
\hline T & Oswaldo Vera \\
\hline U & Alfredo Zea \\
\hline
\end{tabular}

SOURCE : UNT, BOLETIN INFORMATIVO, N 1 , OCTOBRE 2003, P.7

8 Plusieurs faits caractérisent cette nouvelle direction. Le syndicalisme du secteur pétrolier est nettement surreprésenté avec cinq dirigeants syndicaux (A, C, L, R, U), contre seulement deux dans la direction précédente $(9,12)$ et un dans la Coordination nationale suivante $(\mathrm{t})$. Cela peut s'expliquer par la résistance déployée par les salariés du secteur pétrolier pendant l' "insurrection des gérants" de décembre 2002. Un groupe prend une importance particulière, la FBT avec dix représentants dans la nouvelle direction ( $\mathrm{C}, \mathrm{E}$ assassiné depuis, F, H, I, L, M, O, S, T). Cette organisation rassemble alors les partisans hétérogènes du président de la République, élus pour leurs premiers mandats dans des secteurs divers: les entreprises textiles (C, E), le pétrole (L), l'éducation ( $\mathrm{O}, \mathrm{T})$, les télécommunications (I) et les transports (S). D'autres dirigeants sont majoritairement issus de l'extrême-gauche, le trotskisme (C, E, M) ; ou de la Ligue Socialiste, le groupe maoïste (dont est issu l'actuel président Nicolás Maduro) dirigeant de la FBT (L, O, T) ; mais aussi d'anciens partis dominants (H) ou sans appartenance politique avant l'arrivée de Chávez au pouvoir (I). Le démantèlement de la structure bureaucratique de la CTV permet, selon Héctor Lucéna, " aux forces syndicales de gauche, de gagner en marge de manœuvre " (Lucena H., 2007). Cette montée en puissance de la gauche syndicale ne doit pas occulter une composition pragmatique incluant les présidents de grandes fédérations de l'administration publique (Q) ou de l'alimentation (D) dont les itinéraires politiques ne sont pas liés aux secteurs les plus revendicatifs.

9 Toutefois, cette hétérogénéité va nuire à la pérennité de cette centrale. Les clivages se multiplient entre l'autonomie et la dépendance du syndicalisme à l'égard du gouvernement, entre la défense des revendications économiques et celle du processus politique. Une partie de la direction (issue de la classe ouvrière du secteur textile d'une région à l'ouest de Caracas) dont les dirigeants sont liés au courant trotskiste (C, E, N), forme le Courant de Classe Unitaire Révolutionnaire et Autonome (C-CURA). Celui-ci revendique l'autonomie de la centrale syndicale à l'égard du gouvernement. Cette attitude, si elle suscite la méfiance du gouvernement, jouit d'une relative popularité dans la base et promeut un discours d'extrême-gauche dont les origines sociales sont résolument populaires. La polarisation entre le C-CURA et les fractions les plus subordonnées au gouvernement conduit à l'implosion de la centrale. Cet échec peut être illustré par le positionnement actuel de ces dirigeants syndicaux : trois d'entre eux ont rejoint les rangs de l'opposition (C, D, E), deux défendent une vision extrêmement 
critique du chavisme $(\mathrm{H}, \mathrm{N})$, d'autres ont préféré embrasser une carrière politique $(\mathrm{O}, \mathrm{T})$ ou rejoindre l'appareil bureaucratico-industriel de l'État (A, L, S).

\section{La CBST-CCP, institutionnalisation du mouvement syndical chaviste}

10 Après plusieurs années de crise interne au sein de l'UNT, une nouvelle centrale syndicale est fondée le 9 novembre 2011 : la CBST-CCP. Hugo Chávez, après cinq mois de convalescence depuis l'annonce publique de son cancer, salue l'événement dans le premier acte public « du Chávez nouveau ». En l'espace de quelques mois, l'UNT est pratiquement vidée de toute substance. Une Coordination nationale de vingt-cinq personnes est mise en place pour diriger cette nouvelle structure.

Coordination Nationale de la CBST-CCP mise en place en novembre 2011

\begin{tabular}{|l|l|}
\hline & Prénom, Nom \\
\hline a & Osmal Acosta \\
\hline b & Braulio Álvarez \\
\hline c & José Carrasco \\
\hline d & Telémaco Figueroa \\
\hline e & Amada García \\
\hline f & José Gil \\
\hline g & Mercedes Gutiérrez \\
\hline h & José Karias \\
\hline i & Rubén Linares \\
\hline j & Carlos López \\
\hline k & Luz Márquez \\
\hline l & José Mora Siguaraya \\
\hline m & Argenis Moreno \\
\hline o & Julio Moreno Conejo \\
\hline & Manuel Muñoz \\
\hline
\end{tabular}




\begin{tabular}{|c|l|}
\hline $\mathrm{r}$ & Orlando Pérez \\
\hline s & Raúl Pinto \\
\hline $\mathrm{t}$ & Wills Rangel \\
\hline u & Franklin Rondón \\
\hline v & Eglé Sánchez \\
\hline w & Octavio Solórzano \\
\hline x & Francisco Torrealba \\
\hline y & Marco Tulio Díaz \\
\hline
\end{tabular}

SOURCE : CBST-CCP

11 Le premier constat de ce renouvellement est la substitution pratiquement complète de la direction syndicale. En l'espace de huit ans, seuls subsistent quatre anciens membres de la direction précédente de l'UNT (Ff, Il, Qu, Sx). Les autres ont quitté les rangs du chavisme ou ont entamé une carrière en saisissant les opportunités offertes par le gouvernement en mobilisant leurs contacts dans l'administration, l'industrie ou la politique. Le deuxième constat est celui de la consolidation d'un groupe dirigeant à l'affiliation politique homogène. Durant la tutelle d'AD, des dirigeants de partis dominants alliés étaient cooptés (12). Désormais, l'ensemble de la direction syndicale est affilié au même parti politique, le PSUV ; ce dernier est le parti fondé en 2007 par Hugo Chávez qui rassemble l'essentiel des forces soutenant le gouvernement bolivarien.

Trois groupes peuvent être distingués au sein de cette nouvelle centrale. Tout d'abord, un groupe des nouveaux venus intègre le champ syndical, des représentants du secteur des paysans et des pêcheurs (b, c, e, h, n). Ensuite, le groupe de la FBT s'est renforcé et homogénéisé et dispose de dix représentants $(\mathrm{d}, \mathrm{f}, \mathrm{g}, \mathrm{j}, \mathrm{l}, \mathrm{o}, \mathrm{t}, \mathrm{v}, \mathrm{w}, \mathrm{x})$. Enfin, il reste quelques dirigeants ayant exprimé des velléités d'autonomie à l'égard de la FBT à un moment donné de cette période de réajustements politiques et syndicaux (i, k, p, q, r, y).

L'intégration du secteur rural est une nouveauté dans le champ syndical. Il est composé de quatre représentants du secteur paysan $(b, c, e, h)$ et du porte-parole national des pêcheurs (n). L'homogénéité sociologique des représentés est cependant discutable. Le secteur rural regroupe des ouvriers agricoles, mais aussi certains membres des coopératives, des petits propriétaires et des pêcheurs artisanaux. La forme d'organisation dans un syndicat rural représenté dans une confédération ouvrière nationale est un phénomène nouveau. Ces dirigeants sont déjà aux postes de direction au sein des organisations paysannes $(b, e)$ ou des associations civiles de pêcheurs (n). Mais leur articulation avec la classe ouvrière traditionnelle est un fait inédit. Ils n'ont pas, par exemple, connu les querelles intestines de l'UNT. L'autre spécificité de ce groupe est un ancrage au territoire local plus fort. Alors que la plupart des dirigeants syndicaux nationaux sont, du fait de leur responsabilité dans leur fédération ou de leur activité politique, retenus très souvent à Caracas, les responsables des secteurs ruraux 
sont beaucoup plus présents dans leurs régions respectives, à l'Ouest (b), au centreOuest (n), à l'Est (h) et au Sud-Est (e) du pays.

Le secteur de la FBT est désormais le groupe dominant au sein de la CBST-CCP. Depuis 2003, ce groupe s'est homogénéisé. Dès la fin 2004, plusieurs dirigeants de la FBT (C, E, H) sont exclus pour des analyses jugées trop critiques. L'actuelle FBT détient aujourd'hui la présidence $(\mathrm{t})$, trois des sept vice-présidences $(\mathrm{v}, \mathrm{w}, \mathrm{x})$ et la coordination générale (j), soit une majorité de cinq des neuf membres du secrétariat politique, qui demeure par ailleurs la plus haute instance de cette centrale syndicale. Tout porte à croire qu'il existe plusieurs voies qui mènent à la FBT : on retrouve d'anciens membres des partis dominants $(t, v)$, d'anciens membres de partis marxistes-léninistes $(j, o, w)$ ou des primo-militants dont le chavisme est le premier engagement politique $(\mathrm{g}, 1)$. Depuis la fondation de l'UNT, la FBT a favorisé le recyclage d'anciens dirigeants syndicaux des partis qui dominaient la vie politique dans la période avant l'arrivée de Chávez au pouvoir. Une des limites de cette direction en comparaison avec celle d'AD dans la CTV est un faible taux de présence des présidents des grandes fédérations au sein de la FBT. Le président de la fédération pétrolière $(\mathrm{t})$, celui de la santé $(\mathrm{w})$ ou encore celui des télécommunications (l), sont membres de la FBT. Mais les autres ne président que de petites fédérations ou sont confrontés à une concurrence syndicale au sein de leur propre secteur d'activité. Des fédérations puissantes telles que celles du secteur public (u), de l'éducation ( $r$ ), de l'électricité (p) ou la principale fédération de la construction (y), échappent aux militants de la FBT.

15 En effet, il existe toujours un secteur de dirigeants maintenant son indépendance avec la FBT. Cinq syndicalistes (i, p, q, r, y) occupaient encore en 2010 une position de direction au sein de l'UNT, alors que la FBT l'a quitté début 2008. Deux d'entre eux (i, y) faisaient d'ailleurs partie du C-CURA, mais leur courte expérience syndicale et politique ne leur permet pas de proposer une orientation différente. Un autre cadre (r) a été réélu à la tête de la fédération de l'éducation, face à un militant de la FBT (0). Dans plusieurs secteurs comme l'électricité ou la santé, des contradictions perdurent entre des syndicalistes défendant des revendications économiques $(k, p)$ et des dirigeants de la FBT maintenant la priorité à la défense du gouvernement $(\mathrm{g}, \mathrm{w})$. Contrairement à ce qui se passait au moment de l'UNT, ceux-ci ne sont plus articulés entre eux, ils demeurent néanmoins isolés mais présents dans un certain nombre de secteurs.

Il ressort de notre enquête qu'une autre différenciation semble exister au sein de la Coordination nationale de la CBST-CCP entre les membres du secrétariat politique et les autres coordinateurs nationaux placés donc plus bas dans la hiérarchie de l'organisation. Sensiblement du même âge (les deux groupes ont 51 ans en moyenne), les membres du secrétariat politique ont une expérience syndicale plus longue de 9 ans. Ce groupe dirigeant est plus expérimenté, provient davantage de la ville de Caracas (une majorité de ses membres a obtenu son premier mandat dans la région de la capitale alors que les autres coordinateurs nationaux sont issus d'autres régions $d u$ pays et sont donc plus représentatifs de la diversité nationale). Cette plus longue socialisation dans la capitale les a menés aux plus hautes responsabilités syndicales du pays.

17 Nous pouvons distinguer également des cheminements politiques différents. On retrouve dans le secrétariat politique le même nombre de militants d'anciens membres des partis dominants $(t, u, v)$ que de militants historiques des petits partis de la gauche vénézuélienne $(\mathrm{j}, \mathrm{r}, \mathrm{w})$ alors que les autres coordinateurs nationaux ont été nettement 
plus nombreux dans les petits partis de la gauche vénézuélienne (e, i, n, o, s) que dans les anciens partis dominants (p). Ces origines politiques se reflètent dans l'autopositionnement présent sur l'échelle gauche-droite. Lorsqu'on demande aux dirigeants syndicaux de se positionner entre 0 (extrême-gauche) et 10 (extrême-droite), le positionnement moyen des membres du secrétariat politique est de 2,6, contre 1,3 pour les autres coordinateurs nationaux. Les membres du secrétariat politique assortissent cet auto-positionnement de remarques méfiantes à l'égard de l'extrême-gauche :

Ce sont des éléments qui coïncident avec la droite et / ou sont pénétrés par la droite ou pénétrés par les services d'intelligence de l'Empire gringo (j); il ne faut pas être totalement de gauche, radical. Je crois qu'il faut être objectif, il ne faut pas être aveugle, il faut être modéré [...]. La gauche comme la droite ont des extrêmes qui ne sont pas bons (u).

\section{L'inexpérience du syndicalisme chaviste}

18 Mais que ces indicateurs révèlent-t-ils de manière globale ? Premier enseignement, le syndicalisme semble être pour ces dirigeants un moyen d'ascension sociale. Plus de $70 \%$ du groupe interrogé s'affirme d'origine sociale pauvre ou très pauvre. À titre d'exemple, la trajectoire sociale des dirigeants syndicaux ressemble très souvent à celle-ci : "j'ai vécu 24 ans au 23 de Enero (un quartier très populaire de la ville de Caracas), après je suis allé à Caricuao, (un quartier de classe intermédiaire excentré), ensuite je suis passé par Montalbán (une zone résidentielle de classe intermédiaire dans un quartier universitaire) et maintenant je vis au Cafetal (zone de l'Est aisé de la capitale). La manière dont j'ai évolué dans le syndicat a été progressive. J'ai amélioré ma qualité de vie. Je ne suis pas riche, je n'ai pas d'argent, mais je vis bien, je subviens à mes besoins » (D). La ville de Caracas occupe la position dominante du champ syndical au Venezuela. La moitié des dirigeants nationaux ont obtenu leur premier mandat syndical dans la région de la capitale. Deuxième enseignement, l'ensemble des individus sélectionnés a eu à un moment ou à un autre des affiliations politiques. Alain Touraine constate la « subordination du syndicalisme au pouvoir politique » dans l'espace latino-américain (Touraine A., 1988: 267). Cette réalité est particulièrement prononcée au Venezuela dont le «haut degré de politisation» est une des caractéristiques du champ syndical (Ellner S., 1995 : 11).

19 L'étude des trajectoires nous permet de mesurer l'ampleur du renouvellement des directions syndicales. Le renversement de la direction de la CTV par la création de l'UNT est le moment d'une transformation profonde des leaders syndicaux nationaux: plus jeunes, la moyenne d'âge passe de 53 à 47 ans, et moins expérimentés, leur temps de militantisme syndical passe de 27 à 19 ans en moyenne. Leurs origines sociales sont plus modestes : seul un dirigeant de l'UNT (H) déclare provenir des classes moyennes et supérieures, alors que quatre de la CTV affirmaient en être issus $(2,3,6,11)$ et sont aussi moins diplômés : aucun n'a dépassé le niveau de la licence à l'UNT, quatre sont parvenus au-delà de ce titre à la $\operatorname{CTV}(2,8,12,13)$. L'auto-positionnement sur une échelle gauche-droite connaît un net gauchissement (3,4 à la CTV ; 1,5 à l'UNT).

La création de la CBST-CCP succédant à l'UNT à la tête du mouvement ouvrier vénézuélien n'est pas porteuse de changements aussi brutaux mais permet de distinguer l'institutionnalisation du mouvement chaviste et le reflux de ladite «Révolution bolivarienne ». La moyenne d'âge augmente de 47 à 51 ans. Ces dirigeants sont plus âgés mais ne sont pas forcément plus expérimentés. Le temps moyen de 
militantisme syndical des dirigeants de la CBST-CCP est compris entre 15 ans, si l'on ne considère pas les groupes paysans et les associations civiles comme organisations syndicales, et 22 ans, si on accepte cette assimilation. Quoiqu'il en soit, cette durée est proche de celle de la direction de l'UNT en 2003 (19 ans). On constate le nonaccomplissement en 2011 de la génération parvenue au sommet du champ syndical en 2003. Comme nous l'avons vu précédemment, de nombreux cadres syndicaux du chavisme ont changé d'horizons en embrassant une carrière politique, administrative ou industrielle ou en reniant leur sympathie au gouvernement. Les nouveaux dirigeants sont désormais considérés, selon les termes de Bourdieu, comme de la « chair à canon pour combattre les vieux qui, commençant à avoir du capital [...] se servent de ce capital pour contester [...]. Celui qui n'a rien est un inconditionnel ; il a d'autant moins à opposer que l'appareil lui donne beaucoup, à la mesure de son inconditionnalité, et de son néant » (Bourdieu P., 1984 : 54). L'institutionnalisation du chavisme mène à une légère évolution de l'indicateur de l'auto-positionnement sur un axe gauche-droite (1,9 pour la CBST-CCP). Cela ne doit toutefois pas masquer les contradictions en fonction du niveau hiérarchique.

\section{Conclusion}

Cette recomposition conduit à une multiplication des structures syndicales. Celles-ci passent de 2871 en 2001 à 6124 en 2008. Ce doublement du nombre de structures syndicales est concomitant à la stagnation du taux de syndicalisation. Le taux de syndicalisation ne dépasse pas $11 \%$ en juillet $2008^{6}$. Le chavisme a signifié dans un premier temps le moment d'un renouvellement des élites syndicales, le renversement d'une vieille bureaucratie liée au parti social-démocrate et l'accession d'une nouvelle génération de responsables syndicaux, plus jeunes et aux origines sociales plus populaires, aux plus hautes responsabilités. Pourtant, plus d'une décennie après la mise en place de la première centrale chaviste, il est difficile d'entrevoir la consolidation de cette nouvelle génération. Le recyclage d'anciennes élites syndicales permet la constitution d'une direction moins critique à l'égard du gouvernement. Ce qui illustre parfaitement l'institutionnalisation du chavisme. Le rôle de la gauche dans chacune de ces centrales exemplifie le flux et le reflux du processus bolivarien. Dans la CTV, la gauche est constituée par des dirigeants syndicaux aux origines plutôt aisées et n'ayant pas une légitimité forte auprès des travailleurs. Dans la direction de l'UNT, ce secteur est représenté par des dirigeants syndicaux ayant une tradition revendicative dans le secteur textile, des militants trotskistes aux origines populaires jouissant d'une importante légitimité à la base. Au sein de la CBST-CCP, cette fraction de la gauche est isolée dans quelques secteurs par des dirigeants ayant peu d'expérience. Ainsi, le gouvernement chaviste a promu puis contenu une gauche qui proposait une direction alternative. "Le soutien extérieur (en l'occurrence celui du gouvernement) ne peut être acquis que si l'organisation ne met pas en question les formes politiques établies, si elle ne suscite pas de méfiance quant à ses buts de long terme » (Offe C., Wiesenthal H., 1980: 107). Le gouvernement Chávez a soutenu la rénovation des organisations syndicales dans l'objectif de renverser les anciennes directions. La méfiance de l'exécutif à l'égard des revendications d'autonomie syndicale l'a conduit à l'institutionnalisation du mouvement syndical vénézuélien. 


\section{BIBLIOGRAPHIE}

Bourdieu, Pierre, "La délégation et le fétichisme en politique ", Actes de la recherche en sciences sociales, $\mathrm{n}^{\circ}$ 52-53, 1984, p. 49-55.

Ellner, Steve, El sindicalismo en Venezuela en el contexto democrático (1958-1994), Caracas, Ed. Tropykos, 1995.

Gil Yepes, José Antonio, « Opinión pública, socialización política y estabilización del régimen », in Jennifer Mc Coy et David Myers (dir.), Venezuela : Del Pacto de Punto Fijo al Chavismo, Caracas, Los libros de El Nacional, 2007, p.255-288.

Iranzo, Consuelo, « Reflexiones sobre los problemas coyunturales y estructurales del movimiento sindical venezolano » in Mary Ferrero, Chávez y el movimiento sindical en Venezuela, Caracas, Alfadil, 2002, p. 53-74.

Lander, Luis, «La insurrección de los gerentes : PDVSA y el gobierno de Chávez », Revista Venezolana de Economía y Ciencias Sociales, vol. 10, nº 2, 2004, p.13-32.

Lucéna, Héctor, « Le mouvement ouvrier dans la révolution bolivarienne », RISAL, 19 avril 2007. http://risal.collectifs.net/spip.php?page=imprimer\&id_article=2126. Page consultée le 10 janvier 2014.

Offe, Claus, Wiesenthal, Helmuth, « Two Logics of Collective Action : Theoretical Notes on Social Class and Organizational Form » in Maurice Zeitlin (dir.), Political Power and Social Theory, vol.1, Carson, JAI Press Inc., 1980, p. 67-115.

Posado Thomas, « Révolution et recompositions syndicales : le court été de l'autonomie syndicale » in Olivier Folz, Nicole Fourtané, Michèle Guiraud (dir.), Le Venezuela d'Hugo Chávez, bilan de quatorze ans de pouvoir, Nancy, Presses Universitaires de Nancy / Editions Universitaires de Lorraine, 2013, p. 193-220

Touraine, Alain, «Le syndicalisme ouvrier ", La parole et le sang. Politique et société en Amérique latine, Paris, Odile Jacob, 1988, p. 259-293.

UNT, Boletin informativo, $\mathrm{n}^{\circ} 1$, octobre 2003, p. 7.

\section{NOTES}

1. Central Bolivariana Socialista de Trabajadores de la Ciudad el Campo y la Pesca de Venezuela (CBST-CCP).

2. Pour une analyse détaillée du mouvement syndical vénézuélien contemporain voir Posado (2013).

3. Comité de organización Política Electoral Independiente

4. Nous utiliserons cette expression de Luis Lander (Lander L., 2004 : 13-32) pour évoquer la grève des classes supérieures de l'entreprise pétrolière combinée à un arrêt de la production par une partie du patronat prête à paralyser l'économie dans l'intention de renverser le gouvernement.

5. Movimiento Solidaridad Laboral (MSL)

6. Source : Ministère du Pouvoir Populaire pour le Travail et la Sécurité Sociale 


\section{RÉSUMÉS}

Le Venezuela a connu une succession de trois centrales syndicales majoritaires en une décennie, répondant à la polarisation autour du gouvernement Chávez. Cet article propose d'étudier les trajectoires sociales, syndicales et politiques des membres de ces trois directions. La direction de la première centrale syndicale, élue en 2001, est dominée par les militants du parti socialdémocrate, dominant avant Chávez, qui géraient la plupart des grandes fédérations. Après la participation de cette structure à plusieurs tentatives insurrectionnelles, une nouvelle centrale est fondée en 2003 pour regrouper l'ensemble des forces syndicales favorables au gouvernement. Sa direction est hétérogène, incluant des forces syndicales de gauche aux origines populaires et des présidents de grandes fédérations plus conciliants. Cette hétérogénéité aboutit à la fondation d'une nouvelle confédération plus subordonnée au gouvernement en 2011, avec à sa tête un groupe dirigeant plus homogène, recyclant notamment des leaders des anciens partis dominants avant Chávez. Le chavisme a promu un renouvellement profond des élites syndicales avec, dans un premier temps, des dirigeants plus jeunes, moins diplômés, aux origines plus populaires et aux convictions politiques plus radicales, mais l'institutionnalisation de ce mouvement n'a pas permis l'avènement de cette nouvelle génération.

Venezuela has experienced a succession of three majority central trade unions in a decade, responding to the polarization around the Chávez government. This article proposes a study of the social, trade union and political trajectories of the leadership members of the three organizations. The leadership of the first central trade union, elected in 2001, was dominated by the militants of the dominant social democratic party before Chávez, who managed most of the major federations. After this structure was involved in several insurrectionary attempts, a new central union was founded in 2003 to bring together all the trade union forces favorable to the government. Its heterogeneous leadership included grassroots left-wing trade union forces and the more conciliatory presidents of the major federations. This heterogeneity led to the foundation in 2011 of a new confederation that was more subservient to the government, with more homogeneous leadership that brought back some of the leaders of the former parties that dominated before Chávez. Chavismo has promoted a profound renewal of the trade union elites, initially with younger and less educated leaders with more popular origins and more radical political convictions, but the institutionalization of the movement has not allowed the advent of this new generation.

Venezuela ha conocido una sucesión de tres centrales sindicales mayoritarias en una década respondiendo a la polarización alrededor del gobierno de Chávez. Este articulo propone estudiar las trayectorias sociales, sindicales y políticas de los miembros de estas tres direcciones. La dirección de la primera central sindical, electa en 2001, está dominada por militantes del partido social-demócrata dominando, antes del liderazgo de Chávez, la mayor parte de las federaciones. Después de la participación de esta estructura en varios intentos insurreccionales, una nueva central está fundada en 2003 para agrupar el conjunto de las fuerzas sindicales favorables al gobierno. Su dirección es heterogénea, incluyendo fuerzas sindicales de izquierda con orígenes populares y presidentes de grandes federaciones más conciliantes. Esta heterogeneidad lleva a la fundación de una nueva confederación más subordinada al gobierno en 2011, liderada por un grupo más homogéneo reciclando dirigentes de los antiguos partidos dominantes, antes de Chávez. El chavismo ha promovido una profunda renovación de los elites sindicales, con al principio, dirigentes más jóvenes, menos diplomados, con origines más populares y con convicciones políticas más radicales pero la institucionalización de este movimiento no ha permitido la entrada de esta nueva generación. 


\section{INDEX}

Mots-clés : Venezuela, trajectoires syndicales, syndicalisme, renouvellement, institutionnalisation, Hugo Chávez, Révolution bolivarienne.

Palabras claves : Venezuela, trayectorias sindicales, sindicalismo, renovación, institucionalización, Hugo Chávez, Revolución bolivariana

Keywords : Venezuela, trade union trajectories, trade unionism, renewal, institutionalization, Hugo Chávez, Bolivarian revolution.

\section{AUTEUR}

\section{THOMAS POSADO}

thomas.posado@free.fr

Doctorant de Science Politique (5ème année)

Université Paris VIII - CRESPPA - CSU.

Thomas Posado est doctorant en sciences politiques à l'Université Paris-VIII, membre du laboratoire CRESPPA-CSU (UMR 7217), et a été ATER de Science Politique en Université de HauteAlsace (2012-2014). Il travaille sur les recompositions syndicales dans le Venezuela contemporain. Par ailleurs, il est membre fondateur du Groupe d'Études Interdisciplinaires sur le Venezuela (GEIVEN). Il a notamment publié des articles sur la dégradation des conditions de vie dans le Venezuela précédant l'accession de Chávez au pouvoir («Les racines économiques et sociales du nouveau bolivarisme ", in Jean Ortiz (dir.), De Bolívar aux Libertadors d'aujourd'hui : l'Amérique latine insoumise, Biarritz, Ed. Atlantica, 2011), la conjoncture politique du Venezuela (Les Etudes du CERI, 187-188, 2012, avec Olivier Compagnon), sur les tensions entre le chavisme et le mouvement ouvrier (Mouvements, 76, 2013), sur le récit de vie d'un dirigeant syndical (Les Cahiers ALHIM, 26, 2013) ou encore l'histoire du mouvement syndical vénézuélien contemporain («Révolution et recompositions syndicales : le court été de l'autonomie syndicale », in Olivier Folz, Nicole Fourtané, Michèle Guiraud (dir.), Le Venezuela d'Hugo Chávez, bilan de quatorze ans de pouvoir, Presses Universitaires de Nancy, 2013 ; Nuevo Mundo Mundos Nuevos, 2013 ; Alternatives Sud, 21, 2014). Il a en outre coordonné un numéro de revue sur les gauches latinoaméricaines (Recherches Internationales, 93, 2012) et un autre sur le Venezuela contemporain (ContreTemps, 25, 2015). 